\title{
Modelo para el análisis de la quiebra financiera en pymes agroindustriales antioqueñas
}

\author{
Model for risk analysis of financial \\ bankruptcy in agribusiness SMEs of \\ Antioquia - Colombia
}

\section{Modelo para análise de risco de falência}

\section{financeira nas PME agronegócio Antioquia - Colombia}

Marisol Valencia Cárdenas* - Johana Tróchez González** Juan Gabriel Vanegas López*** - Jorge Aníbal Restrepo Morales***

\footnotetext{
Ingeniera industrial. MSc en Estadística. PhD en Ingeniería-Industria y Organizaciones. Docente investigadora, Tecnológico de Antioquia, Medellín, Colombia. Correo electrónico: mvalencia@unal.edu.co

** Candidata a Msc en Estadística, Universidad Nacional de Colombia, Sede Medellín, Colombia. Correo electrónico: jtrochezg@ unal.edu.co

*** Economista. MSc en Economía. Docente investigador en la Fundación Universitaria Autónoma de las Américas. Facultad de Ciencias Económicas y Administrativas, Grupo GICEA, Medellín, Colombia. Correo electrónico: juan.vanegas@uam.edu.co, jg.tecnologico@gmail.com.

**** Ingeniero administrador, Msc en Administración, PhD en Economía de Empresa. Docente investigador Tecnológico de Antioquia Institución Universitaria. Grupo R.E.D. Medellín, Colombia. Correo electrónico: jrestrepo@tdea.edu.co, gifatdea@gmail.com.
} 


\section{Resumen}

El riesgo de quiebra en el sector agroindustrial antioqueño no ha sido ampliamente explorado a partir de modelación estadística, que puede proveer importantes elementos y tendencias para conocer el comportamiento de este sector determinante en la economía regional. Este análisis se basó en un modelo lineal mixto, que utilizó como variable respuesta el puntaje obtenido con el índice $\mathrm{Z}$ de Altman, el cual es un índice latente estimado a partir de variables financieras observables y que, dependiendo de su valor, clasifica las empresas en solventes e insolventes. La metodología utilizada determinó, en primera instancia, el tamaño de muestra por subsector, y luego estimó el índice $\mathrm{Z}$ de Altman por empresa y año, para el periodo comprendido entre 2010-2014. Con dichos índices como respuesta por empresa, se estableció un modelo lineal mixto, que permitió identificar la presencia de cambios significativos entre los diferentes subsectores de la agroindustria antioqueña, para evaluar y diagnosticar problemas de insolvencia futuros para el sector.

Palabras clave: riesgo de quiebra, economía de la agricultura, métodos estadísticos, modelo lineal mixto.

Clasificación JEL: K35, N50, C33 


\section{Abstract}

The risk of bankruptcy in Antioquia's agribusiness sector has not been extensively explored using statistical modeling, which can provide important elements and trends in order to understand the behavior of this important sector for the regional economy. This paper estimated a linear mixed model, which was used as the response variable the score obtained with Altman's Z-Score Model, which is a latent index estimated on the basis of observable financial variables and that depending on its value, ranks companies as solvent or insolvent. The Altman's Index was computed by company and year within the period 2010-2014, using a sample of 214 small and medium-sized companies from different subsectors of the agribusiness sector in Antioquia. The results show that the percentage of firms that enter the area of insolvency increased systematically during the time period from $8.08 \%$ in 2010 to $13 \%$ in 2014 .

Keywords: risk of bankruptcy, agricultural economics, statistical methods, linear mixed model. 


\section{Resumo}

O risco de falência no setor agroindustrial Antioqueño não foi exaustivamente explorado a partir de modelagem estatística, o que pode fornecer elementos e tendências importantes para entender o comportamento deste setor determinante na economia regional. Este trabalho, desenvolveu um modelo baseado em um modelo misto linear, usada como variável resposta a pontuação obtida com o índice $\mathrm{Z}$ de Altman, que é uma estimativa com base em variáveis financeiras observáveis e que dependendo de seu valor, classifica as empresas solventes e insolventes. A metodologia utilizada determinou, na primeira instância, o tamanho da amostra determinada pelo subsetor, em seguida, estimou o índica Z de Altman por empresa e ano, no período compreendido entre 2010-2014. Com esses índices em resposta por empresa, se estabeleceu um modelo linear misto, que permitiu a identificação de alterações significativas entre os diferentes subsetores do Agronegócio Antioqueña, avaliar e diagnosticar problemas de insolvência futura para o setor.

Palavras-chave: Risco de falência, agricultura econômica, métodos estatísticos, modelo linear misto. 


\section{INTRODUCCIÓN}

Las pequeñas y medianas empresas (pymes), así definidas por la Ley 590 de $2000^{1}$ de Colombia, y que según esta, el tamaño de planta de personal y los activos totales son los parámetros que determinan la clasificación, tienen como imperativo mejorar la competitividad para ser sostenibles en el mercado (Restrepo \& Vanegas, 2015). Lo cual se hace cada vez más difícil como consecuencia del aumento de la competencia internacional, producto de la entrada en vigencia de los acuerdos de libre comercio que Colombia pactó con diferentes países, desde el año 2012 con Estados Unidos y en agosto de 2013 con la Unión europea, lo que ha representado un aumento de la competencia local y, a su vez, una oportunidad de emerger en mercados internacionales.

A pesar de que a nivel mundial el sector agroindustrial cobra excesiva importancia, en algunos casos hay inadecuadas tecnologías o problemas de baja competitividad del sector, que hacen de este, un factor vulnerable para la economía de muchos países, en especial, latinoamericanos (García, 2002). El sector primario de otros países, en el que se encuentran subsectores como el de ganadería o de lácteos, han sido afectados negativamente a raíz de la aperturas comerciales; por ejemplo, en México hubo un retroceso en la economía, luego de firmar el TLC de América del Norte (TLCAN), con EU y Canadá en 1994 (Dobson, 2003).

El sector agroindustrial colombiano posee algunas similitudes con respecto al de México, en relación con los problemas acerca de la falta de innovación tecnológica, creación de modelos productivos, la falta de apoyo al pequeño productor, así como la pobreza en las áreas rurales, entre otras. Como consecuencia de estas dificultades, el Gobierno mexicano implementó un programa de subsidios, pero muy bajos frente a los que otorgan otros países como Estados Unidos a sus productores (García, 2002). Por estas razones, debe ser revisada la situación de solvencia financiera del sector agroindustrial colombiano.

Como dice García, (2002), “Con la firma del TLC y la nueva crisis económica mexicana de 1994-1995 se profundiza la crisis de la economía mexicana y crece la pobreza en el campesinado, sin ninguna alternativa en el propio campo, ni tampoco en los otros sectores económicos afectados por la recesión y la creciente inestabilidad financiera”. Dicho estudio se complementa por López (2010), quien analiza la situación de la industria colombiana luego de la apertura económica de la década del 90, y sostiene que es imperante la revisión de las consecuencias y

En Colombia, según la Ley para el Fomento de la Micro, Pequeña y Mediana Empresa, Ley 590 de 2000, las pymes se clasifican así: microempresa: personal no superior a 10 trabajadores. Activos totales inferiores a 501 salarios mínimos mensuales legales vigentes. Pequeña empresa: personal entre 11 y 50 trabajadores. Activos totales mayores a 501 y menores a 5.001 salarios mínimos mensuales legales vigentes. Mediana empresa: personal entre 51 y 200 trabajadores. Activos totales entre 5.001 y 15.000 salarios mínimos mensuales legales vigentes. 
las condiciones actuales de la industria para aumentar su integración al comercio mundial. En dicho estudio se encontró un hecho negativo para Colombia, “...la profundización de esta reforma a partir de la década de 1990 facilitó el aumento inusitado de las importaciones industriales y que esto a su vez agravó el estancamiento del crecimiento industrial y dificultó aún más la transformación y diversificación de la industria". Así mismo, García I. (2002), afirma que "Los efectos de la apertura en la industria se han reflejado, por una parte, en una desaceleración del ritmo de crecimiento en el sector después de 1995". Además, sostiene que se redujeron las áreas cultivadas en la agricultura y se deterioró la competitividad en precios, dado el incremento en las importaciones.

La agricultura ha sido rentable desde hace décadas, pero para algunos países funciona mejor que otros. Contrario a la situación mexicana, gracias a los subsidios en Estados Unidos (García, 2002), o Rusia, este sector sobrevivió. Algunos aspectos que se deben considerar son la larga duración del ciclo de inversión, las condiciones para acceso a los fondos, para evitar que esto dé lugar a una disminución significativa de la producción agrícola y al empobrecimiento de los productores (Iurkova \& Sadovnikova, 2015).

En países como Rusia, actualmente todo ha cambiado radicalmente, gracias a que se han establecido reformas de inversión (Iurkova \& Sadovnikova, 2015). En dicho país, las reformas frente al comercio exte- rior, el apoyo de la industria, el atractivo de las inversiones directas en la agricultura, hacen que se observe una adecuada rentabilidad en el sector agroindustrial. Los inversores comenzaron a cambiar su actitud hacia la industria agraria, como consecuencia del rápido crecimiento de los productos alimenticios a nivel mundial y al aumento de la importancia de la agricultura en general (Iurkova \& Sadovnikova, 2015). Sin embargo, este resultado no se visualiza en algunos países latinoamericanos como México o Colombia.

Colombia lideraba el ranking regional de productividad agroindustrial en la década de los ochenta y los cinco primeros años de los 90, por encima de países como Brasil, Chile, Ecuador y Costa Rica; desafortunadamente el sector ha venido en decadencia desde entonces, como se muestra en López (2010), o en general, el sector industrial, según García I. (2002), así como en las afirmación publicada en la revista Portafolio (Arango, 2015), donde se hace referencia a la agricultura en los siguientes términos: “.....su crecimiento durante el año 2014 ha sido inferior al de la economía (primer trimestre: $6,4 \%$ total vs. $6,1 \%$ del sector; segundo trimestre: $4,3 \%$ vs. $1,6 \%$; tercer trimestre: $4,2 \%$ vs. $3,4 \%$ )". La revista Dinero, por su parte, publicó un análisis de la balanza comercial por sectores, donde señala que en el primer semestre de 2015, las exportaciones también tuvieron una disminución significativa, debido principalmente a la caída de las ventas foráneas de petróleo y derivados $(45,2 \%)$, seguido por una disminución 
de la industria manufacturera $(9,7 \%)$. Las importaciones también se redujeron drásticamente respecto al año 2014, sobre todo en las categorías de alimentos para animales $(-55,25 \%)$, productos alimenticios $(-25,86 \%)$, mientras que los equipos de agricultura y tabaco tuvieron un crecimiento en las importaciones de 73,3 $\%$ y $56,7 \%$, respectivamente. Además, López (2010) revela un estancamiento y decrecimiento de la producción industrial colombiana a raíz de la apertura que inició en 1990. Todo lo anterior muestra cómo el mercado agroindustrial colombiano continúa en decadencia, por ello se hace imprescindible profundizar en el análisis de riesgo financiero de dicho sector.

Las pymes son las que predominan entre la cantidad total de industrias a nivel mundial (Abouzeedan \& Busler, 2004) $\mathrm{y}$, además, estas tiene un amplio campo de investigaciones. Sumado a esto, el comportamiento en ventas y activos de las empresas micro y grandes, cuyo porcentaje es muy bajo, es muy diferente de las pymes. Por eso, la investigación sobre la insolvencia o riesgo de quiebra en el sector agroindustrial ayuda a numerosas industrias e investigadores a fortalecer las decisiones que deben tomarse en este subsector de la economía, para enfrentar las dificultades que pueden estar surgiendo a raíz de tantos cambios económicos.

\section{Estudios de riesgo de quiebra}

En el contexto mundial se han presentado algunos estudios de riesgo relacionados con el sector agroindustrial y el riesgo político y económico. En el país de Birmania, donde el paisaje agrario es el hogar de tres cuartas partes de la población total del país, y se constituye como un potencial sumidero de inversión, primordialmente para la agroindustria nacional e internacional, se han realizado esfuerzos para reposicionar y dar forma proactiva a un entorno jurídico favorable para los negocios que propenda a disminuir los riesgos políticos y económicos y permita que el capital financiero mundial ingrese en los mercados de dicho país de forma más segura, especialmente en el sector agroindustrial (Woods, 2013).

Kornyliuk (2014) presenta un estudio de los principales indicadores de riesgos financieros de las mayores empresas agrícolas de Ucrania, cuyas acciones se negocian en las bolsas de valores extranjeras. Los resultados de la investigación ayudaron a determinar que la liquidez de divisas y cambios de moneda son la mayor amenaza de desarrollo del sector agrícola de dicho país (Kornyliuk, 2014).

Stevanovski, Velkovski, y Stevanovska (2013) estudian las condiciones que permiten determinar efectos sobre las decisiones financieras en el sector agroindustrial de República de Macedonia, y encuentran como factores más significativos el tamaño de las inversiones, el tiempo de los activos que atan, el tamaño del beneficio esperado de ellos y el grado de probabilidad de alcanzar el beneficio esperado. Se presentan distintas posibilidades para la inversión, bajo las condiciones citadas, con algunas combinaciones. 
Restrepo (2014) desarrolla un análisis cuantitativo del riesgo operacional para las pymes en Colombia, donde se calculan las volatilidades de los principales ratios financieros de este tipo de empresas del sector de químicos para el periodo 20002009 , después se utiliza un proceso analítico jerárquico (AHP) para determinar el peso de comparaciones por pares de la rentabilidad, la deuda, la liquidez, los ratios de eficiencia y viabilidad, y establecer un cálculo del riesgo operacional capturando todos los indicadores de la industria, que se convierten en el punto de referencia de evaluación de riesgos. No obstante lo anterior, existen pocos estudios dedicados a la determinación y medición del riesgo inherente a la actividad de la empresa (Celaya \& López, 2013), y en particular en empresas pymes agroindustriales. Tampoco existen estudios estadísticos que permitan estimar modelos longitudinales para probar si existe un detrimento de la solvencia a lo largo del tiempo, en este tipo de empresas del sector agroindustrial antioqueño.

Así como existen numerosos trabajos relacionados con cuantificación del riesgo aplicada a instituciones del sector financiero y la gran empresa (Chernobai \& Rachev, 2006; Rachev, 2006; Chowdhry, 1999; Gillet, Hübner \& Plunus 2010; Frachot, 2003; Cruz, 2004; Marshall \& Marshall, 2001), también es posible encontrar algunos casos aplicados a la pequeña y mediana empresa, como el presentado por Altman y Sabato (2007), quienes desarrollan un modelo de predicción de crisis financiera en pymes americanas con modelos logit combinados con panel de datos; Behr y Güttler (2007) evalúan el riesgo crediticio en pymes alemanas mediante un modelo logit; Fantazzini y Figini (2008) comparan la robustez de modelos longitudinales clásicos y bayesianos en la predicción del riesgo de quiebra; y finalmente, el trabajo reciente presentado por Khemais, Nesrine y Mohamed (2016), modela la predicción de quiebra en pymes tunecinas mediante modelos logísticos y análisis discriminante.

Para el caso de Colombia, el índice Z de Altman se ha utilizado en diversos estudios, por un lado, se evaluó el riesgo de quiebra en algunas empresas del sector manufacturero de Santander, específicamente en la capital Bucaramanga (Marín, 2008), el modelo logístico: una herramienta para evaluar el riesgo de quiebra), donde el sector comercial tiene gran participación en el mercado (32\%). En dicho trabajo, se compara el modelo Z de Altman con un modelo Logit (Marín, 2008). Igualmente, esta metodología fue aplicada para evaluar la solvencia del sector manufacturero de calzado y productos relacionados, y se encontró que el sector en general está en una probabilidad media de riesgo, lo cual amerita que se exploren las causas de dichos problemas, para determinar adecuadamente estrategias de apoyo o mejora (Hernández et al., 2015). Sin embargo, no se encuentran trabajos asociados con modelación longitudinal estadística sobre la insolvencia financiera que es medida con base en este tipo de indicadores, para determinar los efectos en el tiempo de dichos estados. 


\section{Hipótesis propuestas}

Este trabajo tiene como hipótesis central que el sector agroindustrial colombiano, en conjunto, presenta un deterioro paulatino de la solvencia financiera, que se hace más evidente y crítico con el paso del tiempo.

Se busca también si existen subsectores que puntualmente están más afectados que otros a lo largo del tiempo, para probar si existen evidencias de disminuciones significativas del nivel de solvencia en el periodo de análisis.

\section{Metodología y ficha técnica}

Para probar las hipótesis, se analizará el nivel de insolvencia usando el índice $\mathrm{Z}$ de Altman (Altman, 1968), que mide un riesgo de quiebra, para luego determinar los impactos significativos para cada subsector agroindustrial antioqueño a lo largo del tiempo 2010-2014, usando un modelo lineal mixto. El modelo matemático- financiero, diseñado por Altman, permite diagnosticar y predecir con cierta anticipación la insolvencia financiera empresarial generando un índice y clasificándose en varias escalas (Altman, 1968). Inicialmente, el modelo fue propuesto para empresas manufactureras, después fue extendido a todo tipo de empresas (Astorga, 2013).

La metodología utilizada determina, en primera instancia, el tamaño de muestra por subsector con un error del $6 \%$ y un grado de confianza del $95 \%$; después estima el índice $Z$ de Altman por empresa y año, para el periodo comprendido entre los años 2010 hasta 2014. A continuación los valores obtenidos serán usados como variable respuesta en un modelo lineal mixto, que permitirá identificar la presencia de cambios significativos entre los diferentes subsectores de la agroindustria antioqueña en el campo de evaluación y diagnosticar problemas de insolvencia futuros para el sector. Se acota que los factores que causan dichos cambios serán objeto de análisis en trabajos posteriores.

\section{Ficha técnica}

\begin{tabular}{|l|l|}
\hline Población de referencia & \multicolumn{1}{|c|}{1988} \\
\hline Error del tamaño de muestra & \multicolumn{1}{c|}{ - $\%$ \% $\%$} \\
\hline Nivel de confianza & \multicolumn{1}{c|}{214} \\
\hline Tamaño de muestra & \multicolumn{1}{c|}{ 1. Actividades de agricultura } \\
\hline Subsectores & 2. Cultivos de flores y café \\
& 3. Cría de ganado alta calificación \\
& 4. Cría de ganado baja calificación \\
& 5. Banano baja calificación \\
& 6. Banano alta calificación \\
& 7. Comercio al por mayor y al por menor \\
& 8. Aceites, concentrados, panadería \\
& 9. Elaboración de productos lácteos y cárnicos \\
& 10. Actividades de asesoramiento \\
& 11. Avícolas \\
& 12. Alimentos procesados \\
\hline
\end{tabular}




\section{MODELOS ECONOMÉTRICOS PARA LA MEDICIÓN DEL RIESGO FINANCIERO}

\section{Población de estudio y muestra}

El interés es analizar las pyme del sector agroindustrial antioqueño. En Antioquia existen aproximadamente 16800 empresas en total (Cámara de Comercio de Medellín, 2012), de las cuales el 87.6\% corresponde a microempresas, el $8.7 \%$ a pequeñas, el $2.6 \%$ a medianas y el $1.2 \%$ a grandes. Además, el $15 \%$ de las 16800 corresponde a alimentos, bebidas y tabaco (2520 empresas), siendo este el segundo grupo más grande del departamento, seguido de textiles. Alimentos es un sector que viene presentando muchos problemas competitivos y de sostenimiento, por falta de tecnologías, innovación, infraestructura (Baca et al., 2012; Botero, 2005; Tróchez \& Valencia, 2014), en especial, la pequeña y mediana empresa (Montoya, Montoya \& Castellanos, 2010).

Sin embargo, los grupos de empresas: "micro" y "grandes" tienen un comportamiento muy diferente o atípico, en relación con los grupos de "pequeñas" y "medianas" empresas, o pymes, que son más semejantes entre sí. Para evitar problemas sesgo por la presencia de valores extremos y la enorme variabilidad presente en las micro o grandes, se prefiere usar en este estudio las del grupo de las empresas pymes.
De acuerdo con lo anterior, el tamaño muestral se calculó con la fórmula de Neyman, dada en la ecuación [1].

$$
n=\frac{\left(\sum_{h=1}^{L} N_{h} \sqrt{p_{h} g_{h}}\right)^{2}}{N^{2} \frac{B^{2}}{k^{2}}+\sum_{h=1}^{L} N_{h} p_{h} g_{h}}
$$

Con base en un total de empresas agroindustriales obtenidas de la base de datos, 1988, se toman N1=1559 empresas pequeñas, N2 $=429$ medianas, donde $\mathrm{P} 1=\mathrm{N} 1 / \mathrm{N}=0.784, \mathrm{P} 2=\mathrm{N} 2 / \mathrm{N}=0.216$, con esta información y con un error del $6 \%$ $(\mathrm{B}=6 \%$ ), un nivel de confianza del 95 $\%(\mathrm{k}=\mathrm{Z}=1.96)$, se obtiene una muestra de 214 empresas entre pequeñas y medianas, de diferentes sectores del sector agroindustrial antioqueño (bases de Superintendencia de Sociedades).

\section{Índice de Altman}

Para la estimación de dicho índice, se utiliza un análisis discriminante múltiple (MDA) (Altman, 1968), la función discriminante es de la forma:

$$
Z=v_{1} x_{1}+v_{2} x_{2}+\ldots+v_{\mathrm{n}} x_{\mathrm{n}}
$$

Donde $\mathrm{Z}$ es un indice latente estimado a partir de las variables observables, $x_{1}$, $x_{2}, \ldots x_{\mathrm{n}}$, son las variables independientes financieras, $v_{1}, v_{2}, \ldots v_{\mathrm{n}}$, son los coeficientes 
discriminantes, dependiendo del valor de $\mathrm{Z}$ clasifica las empresas en solventes e insolventes.

Altman realizó una versión inicial, sin embargo este modelo solo aplicaba para empresas del sector manufacturero que cotizaban en bolsa; posteriormente hizo otra versión para ser usada en todo tipo de empresas que no cotizan en bolsa (Astorga, 2013). Esta versión corresponde a la ecuación [3], que será usada en este trabajo con la muestra obtenida de 214 empresas.

$$
\begin{gathered}
Z=0.717 x_{1}+0.847 x_{2}+3.107 x_{3}+ \\
0.42 x_{4}+0.998 x_{5}
\end{gathered}
$$

Donde,

$x_{1}$ Capital de trabajo/activo total

$x_{2}$ Utilidades retenidas/activo total

$x_{3}$ Utilidades antes de intereses $\mathrm{e}$ impuestos/activo total

$x_{4}$ Valor del mercado de capital/pasivo total

$x_{5}$ Ventas/activo total

Con dicha ecuación se obtiene una puntuación por empresa, que es usada para el análisis clúster, con el cual se clasifican las industrias (ver Tabla 1). Si $\mathrm{z} \geq 2.9$ la empresa no tendrá problemas de insolvencia en el futuro, si $\mathrm{Z} \leq 1.23$ la empresa tiene altas probabilidades de insolvencia, si $1.24 \leq \mathrm{z} \leq 2.89$ la empresa no se encuentra en una zona bien definida (ver Tabla 1).

Tabla 1. Rangos y clasificación del puntaje de Altman

\begin{tabular}{|c|c|c|}
\hline Código & Calificación & Descripción \\
\hline 1 & Mayor que 2.9 & Solvente \\
2 & Entre 2.9 y 1.23 & Medio \\
3 & Menor q 1.23 & Insolvente \\
\hline
\end{tabular}

Fuente: elaboración propia con información de Altman (1968)

\section{Modelo lineal mixto}

El modelo lineal mixto permite evaluar

$$
\mathrm{y}_{\mathrm{i}}=\mathrm{X}_{\mathrm{i}} \beta+\mathrm{Z}_{\mathrm{i}} \mathrm{b}_{\mathrm{i}}+\varepsilon_{\mathrm{i}}+\operatorname{con} i=1,2 \ldots n
$$

diferentes covariables en el tiempo

y determinar el aporte a la variable respuesta, además tiene en cuenta la correlación existente entre individuos y la que hay en el interior de las medidas de cada individuo. Está definido por medio de la ecuación [4] (West, Welch \& Galecki, 2007). 
Supuestos:

$b_{i} \sim N(0, D)$. iid.

$\varepsilon_{i} \sim N(0, R)$. iid.

\section{RESULTADOS}

Índice de Altman con modelo lineal mixto en empresas agroindustriales

Para el análisis, se hizo una segmentación de las pequeñas y medianas empresas del sector agroindustrial antioqueño, ya que este grupo de empresas son similares entre sí, pero muy diferentes a las microempresas o a las de gran tamaño. Se tomaron en cuenta los subsectores principales considerando la clasificación de la ONU (ONU, 2005). Usando la ecuación [3], se calculó para cada empresa el índice $\mathrm{Z}$ de Altman por año, y sus resultados se aprecian en la Tabla 2.

Tabla 2. Subsectores con el promedio de índice de Altman por año

\begin{tabular}{|c|c|c|c|c|c|c|c|c|c|c|}
\hline $\begin{array}{l}\text { Subsector } \\
\text { agroindustrial }\end{array}$ & $\begin{array}{c}\text { Empre- } \\
\text { sas }\end{array}$ & 2010 & 2011 & 2012 & 2013 & 2014 & $\begin{array}{c}\text { Prome- } \\
\text { dio }\end{array}$ & Mín & Max & $\begin{array}{l}\text { Desvia- } \\
\text { ción }\end{array}$ \\
\hline $\begin{array}{l}\text { Actividades de } \\
\text { agricultura }\end{array}$ & 14 & 2,37 & 1,65 & 1,47 & 1,64 & 2,13 & 1,85 & 1,47 & 2,37 & 0,38 \\
\hline $\begin{array}{l}\text { Cultivos de flores } \\
\text { y café }\end{array}$ & 22 & 2,31 & 2,36 & 2,43 & 2,55 & 2,45 & 2,42 & 2,31 & 2,55 & 0,09 \\
\hline $\begin{array}{l}\text { Cría de ganado } \\
\text { alta calificación }\end{array}$ & 14 & 4,81 & 3,94 & 4,44 & 4,31 & 4,07 & 4,14 & 3,94 & 4,81 & 0,34 \\
\hline $\begin{array}{l}\text { Cría de ganado } \\
\text { baja calificación }\end{array}$ & 24 & 1,24 & 1,32 & 1,38 & 1,37 & 1,43 & 1,64 & 1,24 & 1,43 & 0,07 \\
\hline $\begin{array}{l}\text { Banano baja } \\
\text { calificación }\end{array}$ & 36 & 1,73 & 1,60 & 1,61 & 1,59 & 1,57 & 1,72 & 1,57 & 1,73 & 0,06 \\
\hline $\begin{array}{l}\text { Banano alta } \\
\text { clasificación }\end{array}$ & 26 & 3,32 & 3,44 & 3,53 & 3,54 & 3,51 & 2,97 & 3,32 & 3,54 & 0,09 \\
\hline $\begin{array}{l}\text { Comercio al por } \\
\text { mayor y al por } \\
\text { menor }\end{array}$ & 9 & 3,71 & 2,84 & 2,91 & 2,93 & 2,82 & 3,04 & 2,82 & 3,71 & 0,38 \\
\hline $\begin{array}{l}\text { Aceites, concentra- } \\
\text { dos, panadería }\end{array}$ & 13 & 3,18 & 3,07 & 3,05 & 3,58 & 3,66 & 3,31 & 3,05 & 3,66 & 0,29 \\
\hline $\begin{array}{l}\text { Elaboración de } \\
\text { productos lácteos } \\
\text { y cárnicos }\end{array}$ & 12 & 4,26 & 3,97 & 3,85 & 3,61 & 3,53 & 3,84 & 3,53 & 4,26 & 0,29 \\
\hline $\begin{array}{l}\text { Actividades de } \\
\text { asesoramiento }\end{array}$ & 7 & 2,66 & 2,92 & 2,17 & 2,77 & 2,97 & 2,7 & 2,17 & 2,97 & 0,32 \\
\hline Avícolas & 6 & 2,99 & 3,09 & 2,94 & 3,00 & 3,10 & 3,02 & 2,94 & 3,1 & 0,07 \\
\hline $\begin{array}{l}\text { Alimentos } \\
\text { procesados }\end{array}$ & 31 & 3,34 & 3,46 & 3,25 & 3,74 & 3,38 & 3,43 & 3,25 & 3,74 & 0,19 \\
\hline Promedio total & 214 & 2,78 & 2,64 & 2,58 & 2,74 & 2,68 & 2,69 & 2,58 & 2,78 & 0,08 \\
\hline
\end{tabular}


En la Tabla 2 se aprecia el comportamiento del índice promedio de Altman por subsector: algunos subsectores como cría de ganado baja calificación y banano baja calificación, presentan niveles promedio de 1,64 y 1,72 respectivamente, los cuales tienen dos características importantes, primero han sido muy estables en el tiempo, como lo refleja la desviación estándar de 0,07 y 0,06 respectivamente; y segundo, son muy bajos con alta probabilidad de alcanzar niveles de insolvencia; lo anterior permite inferir que los cambios no responden a eventos inesperados sino que su deterioro ha sido sistemático. Por otro lado, en casos como cría de ganado alta calificación, con 4,14; elaboración de productos lácteos y cárnicos, 3,84; aceites, concentrados, panadería, 3,31; y alimentos procesados, 3,43, se aprecian altos niveles de solvencia financiera y una relativa estabilidad en el tiempo de acuerdo con las desviaciones estándar que exhiben. A pesar de que algunos casos muestran estabilidad en la zona de solvencia, su comportamiento tiene una tendencia a la baja, por ejemplo, en cría de ganado alta calificación, y en elaboración de productos lácteos y cárnicos, en el año 2014 presentan valores inferiores en la serie histórica, que si bien no son preocupantes, deben encender las alarmas debido a que estos sectores han mostrado afectaciones, como lo evidencian algunos trabajos relacionados con la economía del sector (Baca et al., 2012; Botero, 2005; Tróchez \& Valencia, 2014).

De acuerdo con la clasificación expresada en la Tabla 1, se observa cómo el 58,33 $\%$ de los subsectores está en la zona de solvencia y el 41,66 \% está en la zona media. No se presenta ningún subsector en la zona insolvente; pese a que los sectores de cría de ganado baja calificación y banano baja calificación, están en una zona gris, muy cerca de ser insolventes con niveles promedio de 1,64 y 1,72 respectivamente. Se aclara, que aunque el subsector en conjunto no se encuentre en la zona insolvente, es posible que el análisis por empresa muestre algunas unidades en la zona de insolvente, como se mostrará en el análisis posterior en el modelo lineal mixto.

Lo más relevante de la Tabla 2 y que se ilustra en la Figura 1, es la tendencia a disminuir que presenta el sector en conjunto y que se refleja en el nivel medio de dicho índice para el sector. Es posible especular sobre sus causas, y una de ellas quizá pueda residir en la entrada en vigencia de los diferentes tratados de libre comercio que Colombia firmó, pero es un tema que debe ser estudiado más a fondo. 


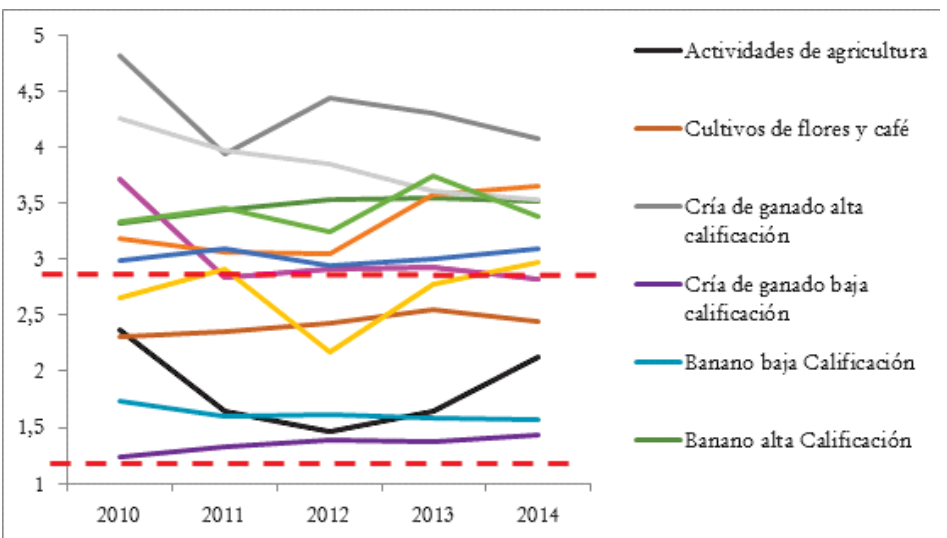

Figura 1. Promedios del índice Z de Altman por subsector

La Figura 1 ilustra las tendencias del promedio del índice $\mathrm{Z}$ de Altman por sector. En esta, las líneas rojas horizontales corresponden a los límites que establecen la división entre solvencia e insolvencia financiera. Se observa como por encima del nivel solvente se ubican subsectores como cría de ganado alta calificación y elaboración de productos lácteos y cárnicos, pero que dibujan tendencias de descenso en el tiempo considerado.

Con la información anterior, se procede a estimar el modelo lineal mixto, donde las covariables exploradas son la clasificación de los subsectores de las empresas (ver
Tabla 2), y la clasificación del índice Z de Altman (Clas_Zm) (ver Tabla 1). Esta variable se ingresa debido a que aumenta la eficiencia del modelo y ayuda a evaluar la significancia del sector.

\section{Clasificación del índice $\mathrm{Z}$ de Altman}

En este apartado, el análisis se escala por unidad empresarial y se usa el mismo modelo propuesto por Altman (1968), para clasificar las empresas en los niveles: solvente, medio e insolvente, de acuerdo con las fronteras definidas en la Tabla 1. Los resultados por grupo y año se muestran en la Tabla 3. 
Tabla 3. Clasificación de la solvencia financiera de las empresas por año

\begin{tabular}{|c|c|c|c|}
\hline Año & Orden & \% Empresas & Calificación (puntaje promedio) \\
\hline \multirow{3}{*}{2010} & Solvente & 35 & 4,50 \\
& Medio & 57 & 2,04 \\
& Insolvente & 8 & 0,46 \\
\hline \multirow{3}{*}{2011} & Solvente & 36 & 4,29 \\
& Medio & 53 & 2,01 \\
& Insolvente & 11 & 0,68 \\
\hline \multirow{3}{*}{2012} & Solvente & 33 & 4,43 \\
& Medio & 54 & 2,00 \\
& Insolvente & 13 & 0,63 \\
\hline \multirow{3}{*}{2013} & Solvente & 34 & 4,58 \\
& Medio & 54 & 2,03 \\
& Insolvente & 12 & 0,65 \\
\hline \multirow{2}{*}{2014} & Solvente & 39 & 4,22 \\
& Medio & 48 & 2,03 \\
& Insolvente & 13 & 0,61 \\
\hline
\end{tabular}

Se aprecia como el porcentaje de empresas que ingresan a la zona de insolvencia, se incrementa de forma paulatina en el horizonte de evaluación, pasando de un $8,08 \%$ en 2010 a un $13 \%$ en 2014. Por su parte, las empresas clasificadas como solventes, en promedio, son el 35,37 \% del sector, las clasificadas en medio son el 53,21\% y las insolventes representan el $11,41 \%$.

\section{Modelo lineal mixto}

El modelo lineal mixto estimado para este trabajo, tiene en cuenta intercepto y pendiente aleatoria, donde la variable respuesta, $\mathrm{Y}_{\mathrm{ij}}$, corresponde al puntaje de Altman en la empresa i, y el año j, con una escala de equivalencia de 2010 a 2014 así: $\mathrm{j}=1,2,3$, 4. En la Tabla 4 se observa la significancia de cada una de las variables: sector y la interacción sector-año, con un nivel del $5 \%$.

Prueba de hipótesis del modelo lineal mixto:

$\mathrm{H}_{0}=$ no hay indicios de variación en la solvencia financiera del sector agroindustrial colombiano en el periodo 2010-2014.

$\mathrm{H}_{\mathrm{a}}=$ hay indicios de variación en la solvencia financiera del sector agroindustrial colombiano en el periodo 2010-2014.

La significancia de la interacción indica que existen cambios evidentes en la solvencia de un subsector a otro, y que avanza por año en el horizonte de tiempo considerado, es decir, financieramente existen subsectores más afectados que otros. 
No obstante la presencia de puntos de residuales desviados, estos son pocos y la mayoría se encuentra en el intervalo $(-3,3)$. Además, la raíz del MSE es 0,6879617 , valor muy pequeño, que indica un ajuste aceptable.

La significancia de la interacción sectoraño, indica que para algunos sectores hubo un crecimiento del índice $\mathrm{Z}$ de Altman, y para otros un decrecimiento, como se aprecia en la Tabla 5, en los valores estimados de los efectos (columna estimación). De esta se puede deducir que los 12 subsectores, 7 que representan el 58,33\% de la muestra, tienen una disminución en la solvencia financiera que resulta significativa, lo que indica un detrimento paulatino de su sostenibilidad económica. Los subsectores que requieren mayor atención son la cría de ganado baja calificación, banano baja calificación, banano alta clasificación, comercio al por mayor y al por menor agroindustrial, elaboración de productos lácteos y cárnicos, y en menor medida el subsector de avícolas y alimentos procesados.

Tabla 4. Significancia de los parámetros del modelo

\begin{tabular}{|c|c|c|c|}
\hline & Chisq & Df & Pr(>Chisq) \\
\hline (Intercept) & 31,5768 & 1 & $1,92 \mathrm{E}-08$ \\
Sector & 105,8706 & 11 & $<2,2 \mathrm{e}-16$ \\
Año & 0,2511 & 1 & 0,6163 \\
Sector: año & 49,1987 & 11 & $8,72 \mathrm{E}-07$ \\
\hline
\end{tabular}


Tabla 5. Valores de efectos parámetros fijos del modelo.

\begin{tabular}{|l|c|c|c|c|}
\hline \multicolumn{1}{|c|}{ Nombre subsector } & Variable & Estimación & Error Std & Valor t \\
\hline & (Intercept) & 1,7937 & 0,3192 & 5,6190 \\
\hline Cultivos de flores y café & sect2 & 0,6263 & 0,4026 & 1,5560 \\
\hline Cría de ganado alta calificación & sect3 & $-0,1543$ & 0,3963 & $-0,3890$ \\
\hline Cría de ganado baja calificación & sect4 & 2,3439 & 0,4433 & 5,2880 \\
\hline Banano baja calificación & sect5 & $-0,0778$ & 0,3724 & $-0,2090$ \\
\hline Banano alta clasificación & sect6 & 1,1350 & 0,3909 & 2,9030 \\
\hline $\begin{array}{l}\text { Comercio al por mayor y al por } \\
\text { menor agroindustrial }\end{array}$ & sect7 & 1,2468 & 0,4991 & 2,4980 \\
\hline Aceites, concentrados, panadería & sect8 & 1,5142 & 0,4514 & 3,3540 \\
\hline $\begin{array}{l}\text { Elaboración de productos lácteos } \\
\text { y cárnicos }\end{array}$ & sect9 & 2,0485 & 0,4607 & 4,4460 \\
\hline $\begin{array}{l}\text { Actividades de asesoramiento } \\
\text { agroindustrial }\end{array}$ & sect10 & 0,9049 & 0,5396 & 1,6770 \\
\hline Avícolas & sect11 & 1,2310 & 0,5680 & 2,1670 \\
\hline Alimentos procesados & sect12 & 1,5708 & 0,3803 & 4,1300 \\
\hline & Año & 0,0477 & 0,0951 & 0,5010 \\
\hline Cultivos de flores y café & sect2:año & 0,0167 & 0,1200 & 0,1390 \\
\hline Cría de ganado alta calificación & sect3:año & 0,2189 & 0,1181 & 1,8530 \\
\hline Cría de ganado baja calificación & sect4:año & $-0,4109$ & 0,1321 & $-3,1100$ \\
\hline Banano baja calificación & sect5:año & $-0,0747$ & 0,1110 & $-0,6730$ \\
\hline Banano alta clasificación & sect6:año & $-0,1355$ & 0,1165 & $-1,1630$ \\
\hline $\begin{array}{l}\text { Comercio al por mayor y al por } \\
\text { menor agroindustrial }\end{array}$ & sect7:año & $-0,2877$ & 0,1487 & $-1,9350$ \\
\hline Aceites, concentrados, panadería & sect8:año & 0,1614 & 0,1345 & 1,2000 \\
\hline $\begin{array}{l}\text { Elaboración de productos lácteos } \\
\text { y cárnicos }\end{array}$ & sect9:año & $-0,3034$ & 0,1373 & $-2,2100$ \\
\hline $\begin{array}{l}\text { Actividades de asesoramiento } \\
\text { agroindustrial }\end{array}$ & sect10:año & 0,0182 & 0,1608 & 0,1130 \\
\hline Avícolas & sect11:año & $-0,0298$ & 0,1693 & $-0,1760$ \\
\hline Alimentos procesados & sect12:año & $-0,0680$ & 0,1133 & $-0,6000$ \\
\hline
\end{tabular}


Es importante anotar como los resultados del modelo lineal mixto se encuentran alineados con los problemas encontrados con el índice Z de Altman, corroborando que los problemas de solvencia financiera son paulatinos y se agudizan con el paso del tiempo

\section{DISCUSIÓN DE RESULTADOS Y CONCLUSIONES}

Se puede afirmar, con adecuada precisión estadística, que el sector agroindustrial colombiano en conjunto, presenta un deterioro paulatino de la solvencia financiera, que se hace más evidente y crítico con el paso del tiempo. Es claro que el modelo de Altman facilita el análisis conjunto de los indicadores financieros para un grupo de empresas, lo que permite definir zonas de riesgo y detectar, mediante una clasificación, zonas de alta probabilidad de riesgo de quiebra. En el caso del sector agroindustrial, existen subsectores que bordean el límite inferior de los niveles de insolvencia financiera, tal es el caso de los subsectores como el banano y el de lácteos, con una baja calificación. De manera exploratoria, con un análisis longitudinal se encuentra que el porcentaje de empresas con problemas de eficiencia financiera tiene una tendencia ascendente con el paso del tiempo, situación que es corroborada con el modelo lineal mixto.

El análisis del modelo lineal mixto, que utilizó como variable explicativa el puntaje obtenido con el índice $\mathrm{Z}$ de Altman, permitió un análisis de efectos por año y sector, mostrando de forma significativa, que independiente de la existencia de empresas y subsectores con altos puntajes de solvencia, el sector en su conjunto evidencia una tendencia negativa en el periodo de análisis, que se agudiza más en la producción de banano, el comercio al por mayor y menor en agroindustria, y la producción de lácteos. Estos hallazgos van en concordancia con la problemática que han visualizado autores como López (2010), quien mostró la desindustrialización o estancamiento a raíz del inicio de las aperturas en 1990 en Colombia, o García I. (2002), o como lo indican los datos encontrados en Portafolio (citado por Arango, 2015), o en la revista Dinero.

Frente a los anterior, resulta paradójico que el sector de banano presente un paulatino deterioro financiero, haciendo imperativo aplicar más modelos de medición y control de riesgo, los cuales inicialmente se desarrollaron para empresas del sector financiero (Basilea II, 2004); pero, mediante su adaptación a empresas de otros sectores de la economía permiten una detección temprana de la insolvencia para desarrollar planes de contingencia que busquen mejorar las capacidades y tecnología del sector, así como sistemas de control y monitoreo de sus procesos y estados financieros, con el propósito de subsanar los riesgos de quiebra oportunamente (Vega, Coronado, Gutiérrez, García \& Díaz, 2006).

Para la economía colombiana, el precio del dólar juega un papel determinante 
desde el punto de vista macro, en el desarrollo y sostenibilidad de las empresas agroindustriales, toda vez que la mayoría participa de forma bilateral, tanto en la compra de insumos como en la comercialización de productos terminados en el exterior (Álvarez, 2015).

Si se buscan mejoras en el sector, pueden fortalecerse las capacidades, las tecnologías y las condiciones para la competitividad en el mercado externo y con ello, aprovechar incrementos en el precio de la divisa, lo que origina a corto y medio plazo, un aumento en el costo de producción, así como también un incremento de las ventas en términos nominales, haciendo más atractivo el proceso de la exportación de bienes, mejorando los ingresos, la rentabilidad y, por tanto, la solvencia financiera. Estos incrementos benefician de forma directa e inmediata a las empresas productoras y exportadoras de sectores como el de flores, café, plátano, azúcar y pescado, al percibir más ingreso en pesos colombianos por su producción. Estos bienes exportables representan aproximadamente el $11 \% \mathrm{del}$ total de las exportaciones de Colombia, por lo que los organismos estatales de apoyo y fomento de la exportación deben tomar estos subsectores como trampolín para impulsar las exportaciones (OCDE, 2015) y el sector agroindustrial.

\section{REFERENCIAS}

Abouzeedan, A. \& Busler, M. (2004). Typology analysis of performance models of small and medium-size enterprises (SMEs). Journal of International Entrepreneurship, 2(1-2), 155-177.

Altman, E. I. (1968). Financial Ratios, Discriminant Analysis and the Prediction of Corporate Bankruptcy. The Journal of Finance, 23(4), 589-609. DOI: http:// dx.doi.org/10.1111/j.1540-6261.1968.tb00843.x

Altman, E. I. \& Sabato, G. (2007). Modelling Credit Risk for SMEs: Evidence from the US Market. Abacus, 43(3), 332-357. DOI: http://dx.doi.org/10.1111/j.14676281.2007.00234.x

Álvarez, C. (2015). Plan de choque. Dinero, 468, 2.

Arango, L. (2015). Agro: lo que pasó en 2014 y perspectivas del 2015. Portafolio, 1. Recuperado de http://www.portafolio.co/economia/agricultura-2014perspectivas-del-2015 
Astorga, A. (2013). Modelos de predicción de la insolvencia empresarial. México DF: Instituto Federal de Especialistas de Concursos Mercantiles (IFECOM).

Baca, J., Mendoza, B., Sánchez, M., Suarez, V., Volpe, I. \& Altamiranda, L. (2012). Desarrollo y competitividad clave fundamental del sector lacteo colombiano. En Tenth LACCEI Latin American and Caribbean Conference for Engineering and Technology, 10. Retrieved from http://www.laccei.org/LACCEI2012-Panama/ RefereedPapers/RP255.pdf

Behr, P. \& Güttler, A. (2007). Credit Risk Assessment and Relationship Lending: An Empirical Analysis of German Small and Medium $\square$ Sized Enterprises. Journal of Small Business Management, 45(2), 194-213. DOI: http://dx.doi.org/10.1111/ j.1540-627X.2007.00209.x

Botero, J. (2005). Estimación del impacto sobre el empleo de los tratados de libre comercio en Colombia: Análisis de equilibrio general computable. Bogotá: Cepal.

Cámara de Comercio de Medellín. (2012). Industria en Antioquia. Un sector que permanece y crece. Recuperado de http://www.camaramedellin.com.co/site/ Portals/0/Documentos/2012/rueda-de-prensa-industria-en-antioquia.pdf

Celaya F, R., \& López P, M. (2013). ¿ Cómo determinar su riesgo empresarial? Revista $\operatorname{EAN}(52), 68-75$.

Chernobai, A. \& Rachev, S. (2006). Applying Robust Methods to Operational Risk Modeling. Journal of Operational Risk, 1(1), 27-41.

Chowdhry, B. \& Howe, J. T. (1999). Corporate Risk Management for Multinational Corporations: Financial and Operational Hedging Policies. European Finance Review, 2(2), 229-246.

Cruz, M. (2004). Operational risk modelling and analys: Theory and practice. London: Incisive Media Investments Limited - Book Risk.

Dinero. (2015, 21 de agosto). La caída de importaciones en junio es menor a la de mayo y abril. Dinero.com. Recuperado de http://www.dinero.com/economia/ articulo/importaciones-junio-2015-dane/212618

Dobson, W. D. (2003). Developments in the Dairy Industries of Mexico, Central America, Argentina, and Brazil--Implications for the US Dairy Sector. Babcock Institute Discussion Paper, (2003-4).

Fantazzini, D. \& Figini, S. (2008). Default Forecasting for Small-Medium Enterprises: Does Heterogeneity Matter? International Journal of Risk Assessment and Management, 11(1-2), 138-163. 
Frachot, A. (2003). Loss Distribution Approach in Practice. Obtenido de http://www. thierry-roncalli.com/download/lda.pdf

García, R. (2002). Crisis agrícola, Tratado de Libre Comercio y migración internacional en México. En Ponencia presentada en el II Congreso Mundial sobre Comercio y Desarrollo Rural, en La Guardia, España (pp. 24-25). Recuperado de www. eumed.net/cursecon/ecolat/

García I., J.G. (2002). Liberalización, cambio estructural y crecimiento económico en Colombia. Cuadernos de Economía, 21(36), 189-244. Recuperado de http://www.scielo.org.co/scielo.php?script=sci_arttext\&pid=S012147722002000100009\&lng=en\&tlng=es.

Gillet, R., Hübner, G. \& Plunus, S. (2010). Operational Risk and Reputation in the Financial Industry. Journal of Banking \& Finance, 34(1), 224-235.

Hernández González, J. W., Daza Castro, N., Moreno, M., Viviana, L., \& Díaz Castro, J. (2015). Aplicación del Modelo Z Score de Altman al sector de manufactura de calzado y productos relacionados en Colombia.

Iurkova, M. \& Sadovnikova, E. (2015). The Main Tendencies of the Development of Russian Agrarian Sector and the Effect of State Policy on Investment Sphere in Agriculture. Poljoprivreda i Sumarstvo, 61(1), 41.

Khemais, Z., Nesrine, D. \& Mohamed, M. (2016). Credit Scoring and Default Risk Prediction: A Comparative Study between Discriminant Analysis \& Logistic Regression. International Journal of Economics and Finance, 8(4), 39.

Kornyliuk, A. (2014). The analysis of the financial risks of domestic agriholdings. The Advanced Science Journal, (11), 65-68.

López, L. F. (2010). Transformación productiva de la industria en Colombia y sus regiones después de la apertura económica. Cuadernos de Economía, 29(53), 239-286.

Marín, J. J. A. (2008). Cómo medir la quiebra de las empresas en Santander, el modelo logístico: una herramienta para evaluar el riesgo de quiebra. REVISTA CIFE

Marshall, C. L., \& Marshall, D. C. (2001). Measuring and managing operational risks in financial institutions: tools, techniques, and other resources. (J. Wiley, Ed.) New York.

Montoya, A., Montoya, I. \& Castellanos, O. (2010). Situación de la competitividad de las pyme en Colombia: elementos actuales y retos. Agronomía Colombiana, 28(1), 107-117. 
OCDE. (2015). Revisión de la OCDE de las políticas agrícolas: Colombia 2015 evaluación y recomendaciones de política. Obtenido de https://www. minagricultura.gov.co/Reportes/OECD_Review_Agriculture_Colombia_2015_ Spanish_Summary.pdf

ONU. (2005). Clasificación industrial internacional uniforme de todas las actividades económicas. Obtenido de http://unstats.un.org/unsd/publication/seriesM/ seriesm_4rev4s.pdf

Rachev, A. C. a. S. (2006). Applying robust methods to operational risk modeling. Journal of Operational Risk, 1, 27-41.

R Core Team. (2014). A Language and Environment for Statistical Computing. Vienna, Austria: R Foundation for Statistical Computing. Retrieved from http://www.rproject.org/

Restrepo, J. (2014). Operative Risk SMES. Global Journal, 65-76.

Restrepo, J. A. \& Vanegas, J. G. (2015). Internacionalización de las pymes: análisis de recursos y capacidades internas mediante lógica difusa. Contaduría y Administración, 60(4).

Stevanovski, M., Velkovski, V. \& Stevanovska, K. (2013). The relation between the long-term investments and the risk in agro-industrial complex. Journal of Hygienic Engineering and Design, 4, 127-131.

Tróchez, J. \& Valencia, M. (2014). Análisis de series temporales en el sector lácteo de Antioquia para detectar efectos de la apertura comercial. Revista Investigaciones Aplicadas, 8(2), 140-151. Recuperado de http://revistas.upb.edu.co/index.php/ investigacionesaplicadas

Vega, S., Coronado, M., Gutiérrez, R., García, A. \& Díaz, G. (2006). Un aporte sobre la industria láctea orgánica y la innovación tecnológica. Revista Mexicana de Agronegocios, 10(19).

West, B., Welch, K. \& Galecki, A. (2007). Linear mixed models A practical guide using Statistical Software. Boca Raton: CRC Press.

Woods, K. (2013). The politics of the emerging agro-industrial complex in Asia's 'final frontier': The war on food sovereignty in Burma. In Paper presented at the Food Sovereignty: A Critical Dialogue. International Conference, Yale University. 